\title{
Application of physical methods in the investigations of crystal-related arthropathies
}

\author{
J. S. S H A H \\ From the H. H. Wills Physics Laboratory, University of Bristol
}

\section{Introduction}

The application of physical methods to understanding the role of crystals in pathogenesis of various arthropathies can help in two ways. Firstly, in exploration of the nature of crystalline deposits-for example, identification of crystals--and, secondly, in understanding the very processes of crystal growth (and dissolution).

The assessment of the crystals mainly comprises:

(1) Determination of chemical composition and defects such as non-stoichiometry and foreign phase precipitation.

(2) Identification of crystal structure and determination of crystallographic parameters.

(3) Visualisation of structural imperfections.

(4) Examination of morphology for determination of conditions of crystal growth. (Finer structural details on a crystalline surface are often associated with the growth history of the crystal.)

Physical methods available for crystal characterisation can be broadly divided into two categories.

(1) Methods based on the interaction of crystals with electromagnetic radiation-for example, visible light, infrared radiation, $x$-rays. These are: (a) polarisation light microscopy; (b) infrared absorption photometry; (c) $x$-ray diffraction methods, including topographic method; and (d) $x$-ray extended absorption free structure (EXAFS).

(2) Methods based on material-electron interaction:

(a) transmission electron microscopy (TEM); (b) scanning transmission electron microscopy (STEM); (c) analytical electron microscopyenergy dispersive $x$-ray analysis (EDX) and wavelength dispersive analysis (WDX); and (d) electron diffraction.
Detailed description of each of the methods is beyond the scope of this paper. Here I intend to deal briefly with the subjects of infrared absorption spectrometry, $x$-ray diffraction, EXAFS, and electron microscopies, and show how they have been applied to investigate arthropathic crystallisation. Where appropriate, examples of other biological and non-biological crystals will be cited.

\section{Methods}

IN FRARED SPECTROMETRY

Vibrations of molecular bonds in a material cause characteristic absorption of the infrared region of the electromagnetic spectrum. The absorption for each bond occurs at specific frequencies, which may reveal the nature of vibrations and stereochemistry of the molecular groups in question. The characteristic peaks may be used like fingerprints to determine the presence of different molecular groups in a substance.

Absorption spectra of apatite and calcium pyrophosphate crystals are different. They may be used to detect the presence or otherwise of the compounds in arthropathic deposits.

In the case of hydroxyapatite infrared spectra may yield additional information on crystallite size. Fig. 1 shows absorption spectra of apatite from calcific periarthritis. The apatite is finely divided. Its crystallite size from line broadening measurements in $x$-ray powder diffraction line was about $50 \mathrm{~nm} .{ }^{1}$ Fig. 1a shows that the peaks at 3572 wavelengths $/ \mathrm{cm}$ are missing in the range of the human deposit but are present in that of synthetic crystals of about $1 \mu \mathrm{m} .^{3}$ The absence of the above peaks in the spectrum of the finely divided apatite is due to the fact that the stretching and bending modes of $\mathrm{OH}$ bonds are

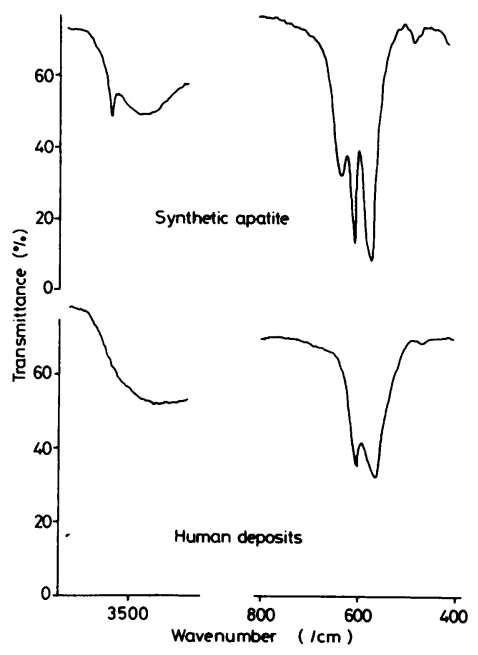

Fig. 1 Infrared spectra of (a) finely divided apatite from periarthritic deposits and (b) Durango hyproxyapatite. Note that peaks at $3572 \mathrm{~cm}$ and $620 \mathrm{~cm}$ are absent in spectrum of human deposits.

perturbed by the $\mathrm{H}$ bonding of water to surface $\mathrm{OH}$ ions of the apatite. ${ }^{2}$

Infrared absorption spectrometry has also been used to show the presence of $\mathrm{CO}_{3}^{2}$ ions in human deposits but I shall refer to this in conjunction with the $x$-ray diffraction results.

\section{X-RAY DIFFRACTION}

Whenever an $x$-ray beam is incident on a crystal it is scattered by the atoms in the crystal. The atoms in the crystals are arranged in a regular manner with a definite periodicity, allowing identification of a series of rows of planes with a constant interplanner spacing. Due to this geometry of the atoms, scattered $x$-rays, when superimposed on each other, are 
annulled except in certain directions where there is a constructive reinforcement. This phenomena is called diffraction. The direction of a diffracted beam can be found by Bragg's law:

$$
\mathrm{n} \lambda=2 \mathrm{~d} \sin \theta,
$$

where $\mathrm{n}=$ order of diffraction $(\mathrm{n}=$ integer) $\lambda=$ wavelength of $x$-rays $d=$ interplanar spacing (characteristic of the crystal lattice) and $\theta=$ angle of diffraction.

Experimentally, one can use $x$-rays of known wavelength $\lambda$, measure angle $\theta$, and hence determine the interplanar spacing $d$. Because biological crystals are very small one uses the powder diffraction method. In practice, powdered specimen is filled in a thin tube or coated on the surface of a very thin glass rod (about $1 \mathrm{~mm}$ in diameter) and mounted in a Debye-Scherrer camera. A film is then placed in the camera. This surrounds the specimen to record diffracted beams as arcs. Such a set of lines is unique to the crystalline substance in question and may therefore be used as the 'fingerprint' of the substance. From interplanar spacings one can evaluate lattice constants of the crystal structure with a great accuracy.

Both hydroxyapatite and calcium pyrophosphate have an immensely complex growth system. ${ }^{1-4}$ Many different phosphate salts can grow from the same reaction system. - Powder diffraction methods, therefore, are immensely useful in determining crystal growth conditions in in vitro systems. Fig. 2 shows diffractographs of (pyrophosphate) crystals grown from solution and gel and that of a deposit from articular cartilage. From these patterns it was deduced that other compounds such as calcium pyrophosphate tetrahydrate (CPPT) can grow in in vitro systems. Pathological deposits have been shown to contain only the mixture of monoclinic and triclinic phases of calcium pyrophosphate dihydrate (CPPD). Because of the fingerprint nature of diffraction lines it is possible to detect as little as $0.1 \%$ weight of the calcium phosphate in another.

Careful determination of lattice parameters allows detection of foreign atoms (impurity) incorporated into the host lattice. Several ions such as $\mathrm{F}^{-} \mathrm{Cl}^{-}$ $\mathrm{CO}_{3}^{2-}$ may be accommodated substitutionally, in hydroxyapatite

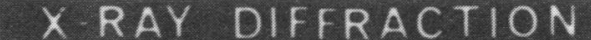

CARTILAGF
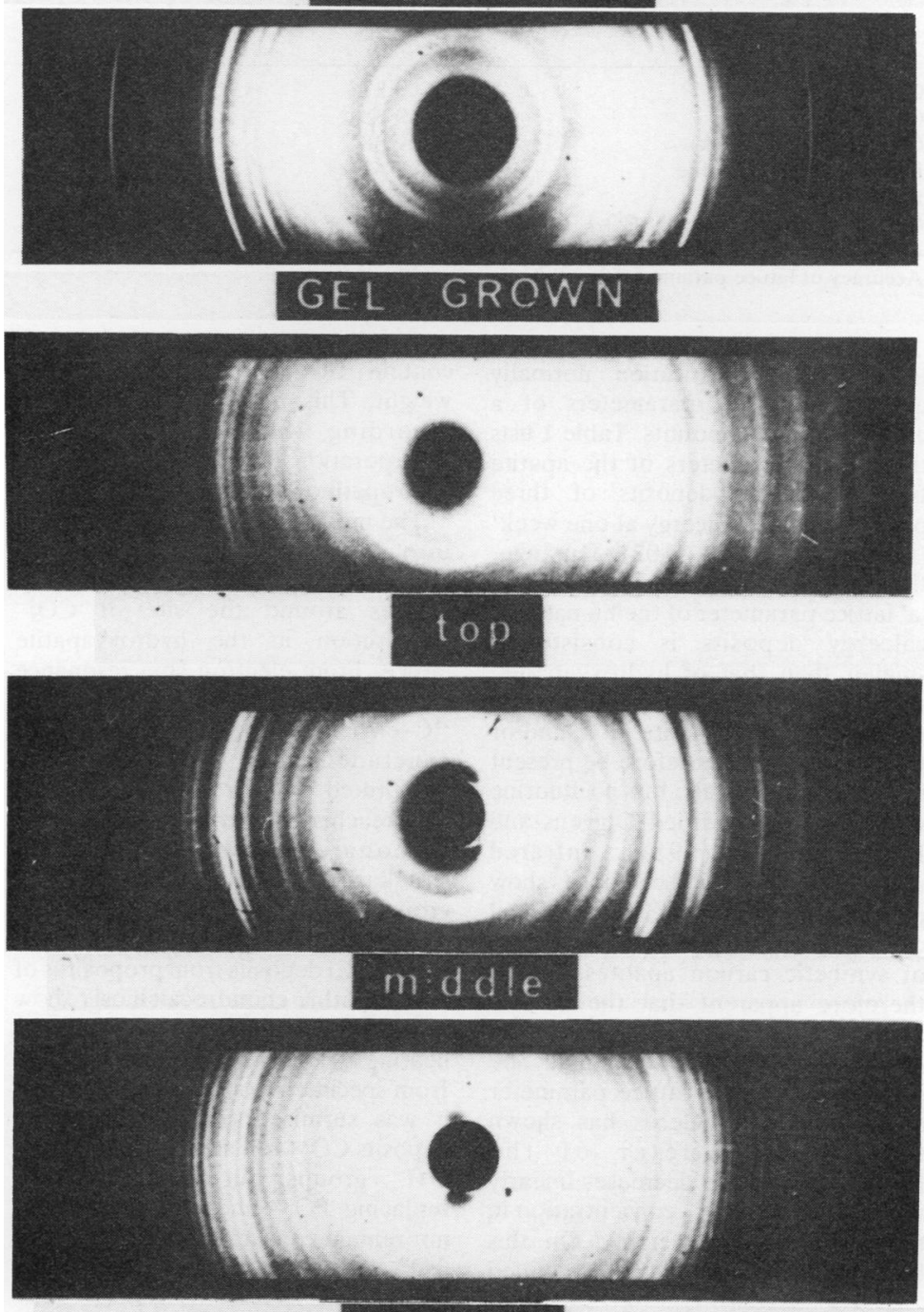

bottom
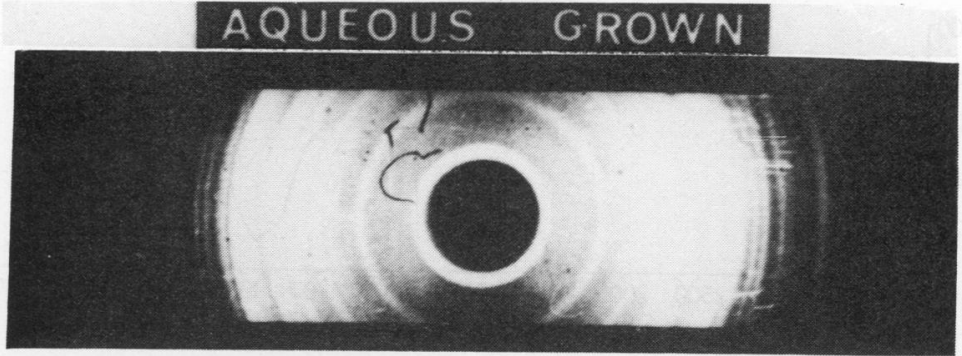

Fig. 2 X-ray powder diffractographs of pyrophosphates: (a) grown from solution (b) grown in gelatin gel, and (c) found on cartilage surface. 
Table 1 Lattice parameters of apatite from particular deposits from three patients

\begin{tabular}{lll}
\hline Sources of apatite & \multicolumn{2}{l}{ Lattice parameters $(\AA)$} \\
\cline { 2 - 3 } & $' a{ }^{\prime}$ & $' c '$ \\
\hline Case 1 & $9 \cdot 37$ & $6 \cdot 88$ \\
Case 2 & $9 \cdot 38$ & $6 \cdot 87$ \\
Calcergy (at one week) & $9 \cdot 38$ & $6 \cdot 88$ \\
Durango hydroxyapatite & $9 \cdot 42$ & $6 \cdot 88$ \\
Fuorapatite & $9 \cdot 37$ & $6 \cdot 88$ \\
Carbon apatite (7\% weight $\left.\mathrm{CO}_{3}\right)$ & $9 \cdot 38$ & $6 \cdot 89$ \\
\hline
\end{tabular}

Accuracy of lattice parameters is $\pm 0.01 \%$.

lattice. Such substitution normally alters the lattice parameters of a crystal by small amounts. Table 1 lists the lattice parameters of the apatite from particular deposits of three patients, simple calcergy at one week ${ }^{1}$ (Harries et al., p. 102) Durango, fluorapatite and carbon apatite. The 'a' lattice parameter of the human and calcergy deposits is consistently smaller than that of hydroxyapatite, and similar to those of both carbon apatite and fluorapatite. $\mathrm{F}^{-1}$ and/or $\mathrm{CO}_{3}^{2-}$ ions could therefore be present in the above crystals, but no fluorine was found by modified Diggens and Rosse method (1981). Infrared spectra (Fig. 3) of all the deposits show peaks at $1460 \mathrm{~cm}, 1415 \mathrm{~cm}$, and 869 $\mathrm{cm}$. These bands appear in the spectra of synthetic carbon apatites. ${ }^{56}$ It is therefore apparent that the in vivo deposits contain carbon apatite. The amount of $\mathrm{CO}_{3}^{2-}$ content can in fact be estimated from the lattice parameter measurements. LeGeros has shown that ' $a$ ' parameter of the hydroxyapatite cell decreases linearly with increasing $\mathrm{CO}_{3}^{2-}$ concentration in the range $0-24 \%$ weight. ${ }^{7}$ On this basis human deposits we examined contain $\mathrm{CO}_{3}^{2-}$ in the range $8-9 \%$ weight. This finding raises question regarding the source of $\mathrm{CO}_{3}^{2-}$ incorporation in in vivo mechanisms of arthropathic apatite growth.

The mode of carbonate substitution into biological apatite remains controversial..$^{5}$ The controversy centres around the site of $\mathrm{CO}_{3}^{2-}$ substitution in the hydroxyapatite lattice. From electron spin resonance and the infrared studies of ${ }^{13} \mathrm{C}$-enriched apatites Doi et al. concluded that $\mathrm{CO}_{3}^{2-}$ ions are substituted at $\mathrm{PO}_{4}^{3-}$ sites. ${ }^{6}$ LeGeros also reached this conclusion because carbonate substitution causes shrinkage in ' $a$ ' parameter of the $\mathrm{CO}_{3}^{2-}$ group. ${ }^{7}$ Matalon and DeBenyacer, however, claim that carbonate content of calcified deposits from propositus of an idiopathic chondrocalcinosis show changes in carbonate content on heating. ${ }^{8}$ This change was variable from specimen to specimen. From this it was surmised that in the above deposits $\mathrm{CO}_{3}^{2-}$ replaces both $\mathrm{PO}_{4}^{3-}$ and $\mathrm{OH}^{-}$groups but that the ratio replacing $\mathrm{PO}_{4}^{3-}$ and $\mathrm{OH}$ group does not remain constant. The exact site of $\mathrm{CO}_{3}^{2-}$ substitution in arthropathic deposits cannot be easily determined by the existing data on infrared absorption and lattice parameter.

APPLICATION OF SYNCHROTRON X RADIATION

Intensities of diffracted beams are very small. In a laboratory, therefore, with normal $x$-ray tube sources it requires about 48 hours to obtain a powder diffraction pattern of a well-crystallised specimen-which contains a few tens of milligrams. It is therefore often impossible to obtain a diffraction pattern of minute deposits in situ-say on a cartilage surface. Recently, however, it has become possible to use synchrotron radiation source, which provides 'tunable' $x$-rays of very high intensity, the diffraction (Fig. 4) pattern of crystalline deposits on cartilage surface. The pattern covers the diffraction angle in the range $\theta=1^{\circ}-12^{\circ}$. This opens up exciting prospects for studying in situ arthropathic deposits. It is concluded (Fig. 4) that crystallites on cartilage show no preferred orientation as they do in bone and enamel.

\section{EX A F S}

This is really a form of $x$-ray absorption spectroscopy. An absorption spectrum of a substance is obtained by shining an intense $x$-ray beam-for example, that from synchrotron source-on it. The spectrum arises because $x$-ray absorption cross section for the excitation of an electron from a deep core of the atom exhibits oscillation as a function of $x$-ray (photon) energy.

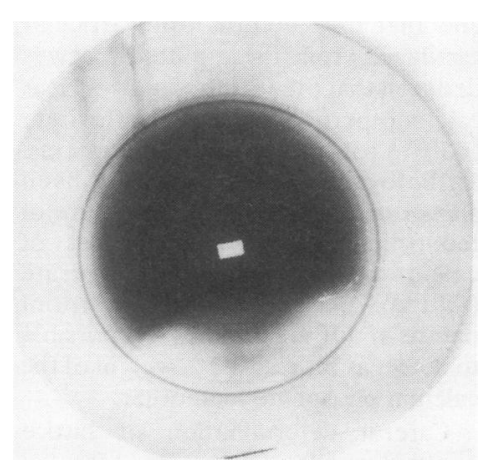

Fig. 4 X-ray diffraction pattern of in situ deposits on articular cartilage surface taken with synchrotron radiation source.
Fig. 3 Infrared spectra of a typical periarthritic deposit. Peaks at $1460 \mathrm{~cm}, 1415$ $\mathrm{cm}$, and $869 \mathrm{~cm}$ are additional to those in the pure hydroxyapatite spectrum. 


\section{Application of physical methods Suppl p 71}

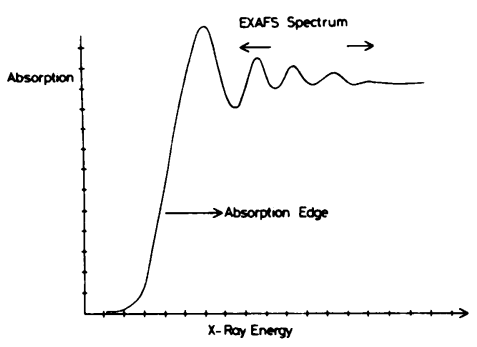

Fig. 5 A schematic diagram of an EXAFS spectrum.

This manifests itself as a fine structure in the $x$-ray absorption spectrum just above the absorption edge (Fig. 5). In contrast to $x$-ray diffraction, EXAFS studies are restricted to the immediate vicinity of the absorber element. They therefore yield information on the local structure and chemical states of bonding sites. On interatomic distances co-ordinate number and geometry of an atom within a complex organic matrix can therefore be studied.

So far EXAFS has not been used to obtain information on arthropathic deposits. Miller et al. and Binstead et al. have applied EXAFS to bone mineral ${ }^{910}$ and have concluded that the calcium 'environment' in bone mineral is not the same as synthetic and geological hydroxyapatite. Instead the bone mineral is shown to have a structure that is intermediate between amorphous calcium phosphate and crystalline hydroxyapatite with high defect density. EXAFS has also shown that in development of bone the calcium environment changes in a similar manner to that of in vitro maturation of amorphous calcium phosphate to poorly crystallised hydroxyapatite.

\section{METHODS BASED ON ELECTRON MICROSCOPY}

When a focused-high energyelectron probe makes an impact with a material it generates a variety of different signals.

Low energy secondary electrons (energy $<50 \mathrm{eV}$ ) generated from near the surface of a material gives information on topography and is used in scanning electron microscopy (SEM). Elastically transmitted electrons are capable of diffraction and give information on structure. The

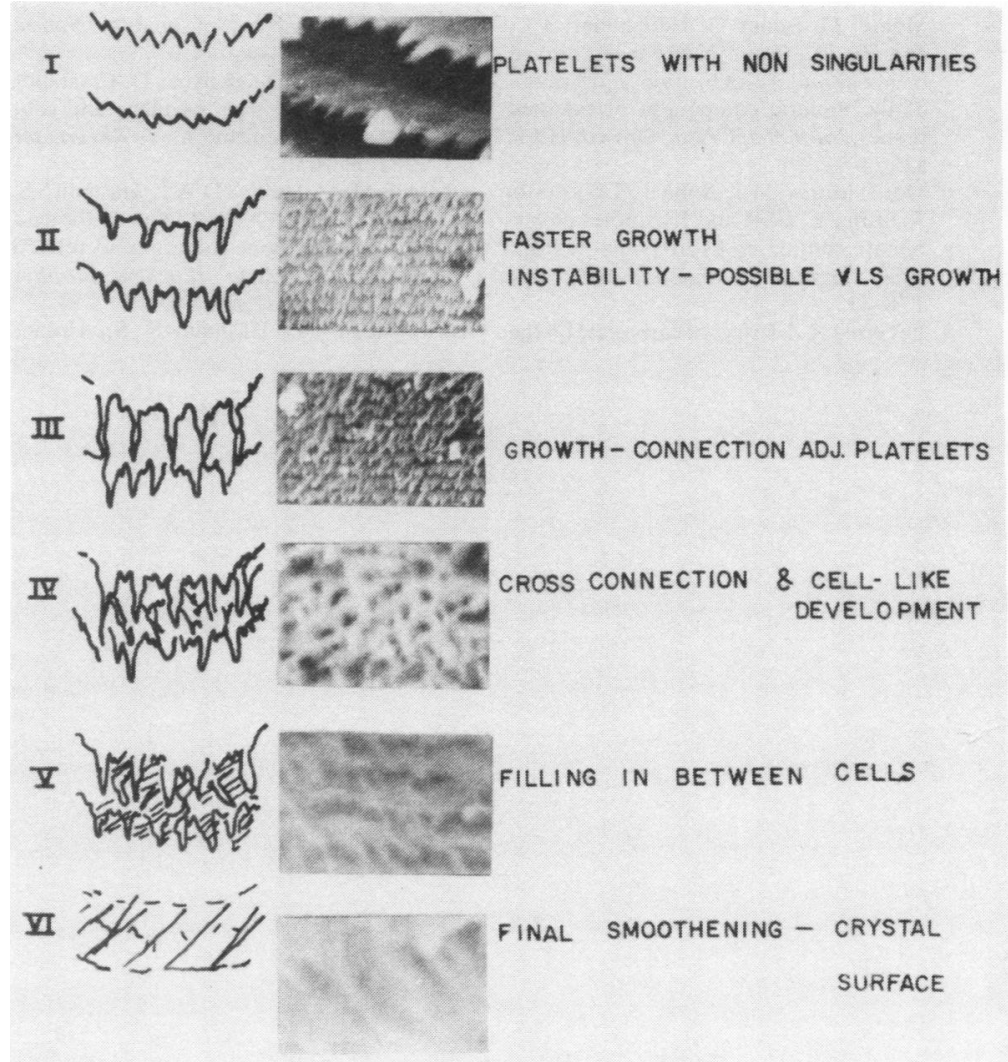

Fig. 6 A sequence of events in the growth of $\mathrm{CuAlS_{2 }}$ crystals grown by chemical vapour deposition techniques.

collision of electrons within the material gives rise to the emission of characteristic $x$-ray radiation which are used in EDX and WDX to give information on chemical composition. With the aid of modern microscopes very high resolution ( $2 \AA$ in TEM and $50 \AA$ in SEM) images can be obtained. The values of these techniques hardly needs to be emphasised. The EDX technique allows construction composition maps with a resolution of $1 \mu \mathrm{m}^{2}$ and has shown that the calcified lesions in simple calcergy have layers and that the $\mathrm{Ca}: \mathrm{P}$ ratio in each layer is different. ${ }^{1}$

So far high resolution microscopy has thrown little light on growth mechanisms of crystals in human arthropathic deposits.

SEM, however, has been used to evaluate a sequence of events (Fig. 6) in the growth of tiny crystals of $\mathrm{CuAlS}_{2}$ grown by chemical vapour deposition technique. ${ }^{11}$
In conclusion, I should like to emphasise that an integrated programme of investigation combining the above methods is likely to contribute in the understanding of the crystal deposition processes in arthropathies.

\section{References}

1 Shah J S, Harries J E, Dieppe P, Heap P. Characterisation of calcium phosphate crystals relevant to crystal deposition diseases. Ann Rheum Dis 1982; 41: 312.

2 Posner A S, Betts F, Blumenthal N C. Formation and structure of synthetic and bone hydroxyapatite. Progress in Crystal Growth and Characterisation 1980; 3: 47-64.

3 Shah J S, Dieppe P. Crystal deposition diseases of the joints. Progress in Crystal Growth and Characterisation 1980; 3: 17-47. of calcium pyrophosphate dihydrate crystals in vivo and vitro. $J$ Rheumatol 1981; 8: 1016.
4 Shah J S, Heap P, Dieppe P. Deposition 
5 Montel G, Bones G, Heughbaert J C, Trombe J C, Rey C. New concepts in the composition crystallization and growth of the mineral component of calcified tissues. Journal of Crystal Growth 1981; 53: 74-99.

6 Doi J, Moriwaki J, Aoba T, Takashashi J, Joshin K. ESR and IR studies of carbonate containing hydroxyapatite. Calcified Tissue International 1982; 34: 178-81.

7 LeGeros R Z. Effect of carbonate on the lattice parameters of apatite. Nature 1965; 206: 403-4.

8 Matalon J R, De Benycar D. Crystallochemical study from cartilage and subcutaneous calcification. $J$ Rheumatol 1981; 8: 1010.

9 Miller R M, Hukins D W L, Hasnain S S, Lagarde P. EXAFS studies of the calcium ions in bone mineral and related calcium phosphate. Biochem Biophys Res Comm 1981; 99: 102-6.

10 Binstead $\mathrm{H}$, Hasnain S S, Hukins
D W L. Developmental changes in bone mineral structure demonstrated by EXAFS spectroscopy. Biochem Biophys Res Commun 1982; 107: 89-92.

11 Shah J S. Growth morphology and impurity characterisation of some I, II, $\mathrm{VI}_{2}$ sulphides and selenides. Progress in Crystal Growth and Characterisation 1981; 3: 333-89. 